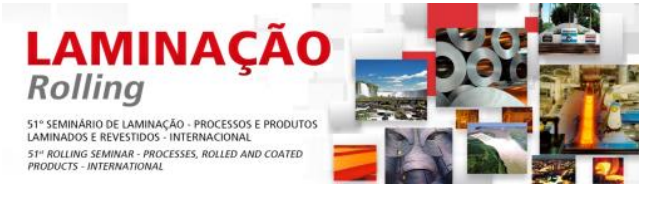

\title{
INFLUÊNCIA DO TEOR DE ALUMÍNIO NO BANHO DE ZINCO NA QUALIDADE SUPERFICIAL DE REVESTIMENTOS GALVANNEALED*
}

\author{
Sandra Goulart-Santos ${ }^{1}$ \\ Aldo Henrique de Almeida Barbosa ${ }^{2}$
}

\section{Resumo}

Os aços galvanizados são amplamente utilizados devido à sua excelente resistência à corrosão. Além disso, esses aços apresentam alta qualidade superficial, o que é um requisito importante para aplicações exigentes como as da indústria automobilística. Dentre os parâmetros de processo importantes para a qualidade dos revestimentos está o teor de alumínio no banho de zinco. Nesse estudo, foi utilizado o simulador do processo de galvanização a quente (HDPS), para investigar a influência do teor de alumínio efetivo do banho na qualidade superficial do revestimento galvannealed (GA), sendo avaliados os teores de $0,09 \%$ e $0,14 \%$. Para acompanhar os estágios de formação das fases Fe-Zn foram realizadas interrupções do ciclo de galvanização ao longo das etapas de imersão no banho e de tratamento térmico de galvannealing. Utilizando a técnica GDOES, foi possível acompanhar as variações da concentração de alumínio ao longo do revestimento durante a formação das fases Fe-Zn. Os resultados das simulações mostraram que o alumínio efetivo mais baixo no banho favoreceu a cinética de formação das fases Fe-Zn durante o tratamento térmico e diminuiu a ocorrência de regiões com aspecto brilhante no revestimento, melhorando a qualidade superficial.

Palavras-chave: Revestimento GA; Qualidade superficial; Alumínio efetivo; GDOES.

\section{INFLUENCE OF THE ZINC BATH ALUMINUM CONTENT ON THE SURFACE QUALITY OF GALVANNEALED COATINGS}

\section{Abstract}

Galvannealed steels are widely used due to its excellent corrosion resistance. In addition, these steels have high surface quality, which is an important requirement for demanding applications such as in the automotive industry. An important parameter for the quality of galvannealed steel is the aluminum content in the zinc bath. In this study, a hot-dip process simulator (HDPS) was used to investigate the influence of aluminum content in the bath on the surface quality of the galvannealed coating; baths with $0.09 \%$ and $0.14 \%$ effective aluminum were evaluated. To follow the formation of Fe-Zn phases, galvanizing cycles were interrupted along the steps of immersion in the bath and heat treatment. Using the GDOES, it was possible to follow the variations of aluminum concentration within the coating during the formation of the phases. The simulation results showed that the lowest effective aluminum in the bath have favored the kinetics of $\mathrm{Zn}$-Fe phases formation during heat treatment and have decreased the occurrence of regions with bright appearance on the coating, improving the surface quality.

Keywords: Galvannealed coating; Surface quality; Effective aluminum; GDOES.

1 Engenheira Química, Doutora em Engenharia Metalúrgica e de Materiais, Pesquisadora do Centro de Tecnologia Usiminas, Ipatinga, MG, Brasil.

2 Engenheiro Metalurgista, Doutor em Engenharia Metalúrgica e de Materiais, Pesquisador Especialista do Centro de Tecnologia Usiminas, Ipatinga, MG, Brasil.

\footnotetext{
* Contribuição técnica ao $51^{\circ}$ Seminário de Laminação - Processos e Produtos Laminados e
} Revestidos, 28 a 31 de outubro de 2014, Foz do Iguaçu, PR, Brasil. 


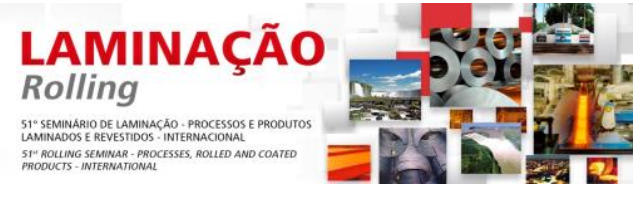

Tabela 1. Composição química dos banhos de zinco via ICP

\begin{tabular}{cccc} 
Banho & $\% \mathrm{Fe}$ & $\% \mathrm{Al}$ & $\%$ Al_ef \\
1 & 0,03 & 0,12 & 0,09 \\
2 & 0,02 & 0,16 & 0,14 \\
\hline
\end{tabular}

Como substrato, foi utilizado um aço bake hardenable com limite de escoamento da ordem de $180 \mathrm{MPa}$. Os ciclos térmicos de galvanização (Figura 1a) consistiram das etapas de recozimento, imersão no banho de zinco, aquecimento indutivo até a temperatura de $550^{\circ} \mathrm{C}$ (temperatura de galvannealing), seguido de um resfriamento brando, com uma taxa correspondente à de resfriamento natural (ao ar livre) e outro, mais acentuado, com taxas superiores a $15^{\circ} \mathrm{C} / \mathrm{s}$. Para acompanhar os estágios de formação das fases Fe-Zn no revestimento, foram realizadas interrupções do processo de galvanização ao longo das etapas de imersão no pote e de tratamento térmico de galvannealing, nos pontos: (I) início da imersão no pote; (II) início do tratamento térmico; (III) após $10 \mathrm{~s}$ de tratamento térmico (Figura 1b) e (IV) após o ciclo completo de produção do revestimento (Figura 1a). Os revestimentos foram resfriados rapidamente com gás hélio (aproximadamente $80^{\circ} \mathrm{C} / \mathrm{s}$ ) para congelar a microestrutura, o que permitiu acompanhar a evolução da formação das fases Fe-Zn ao longo do processo.

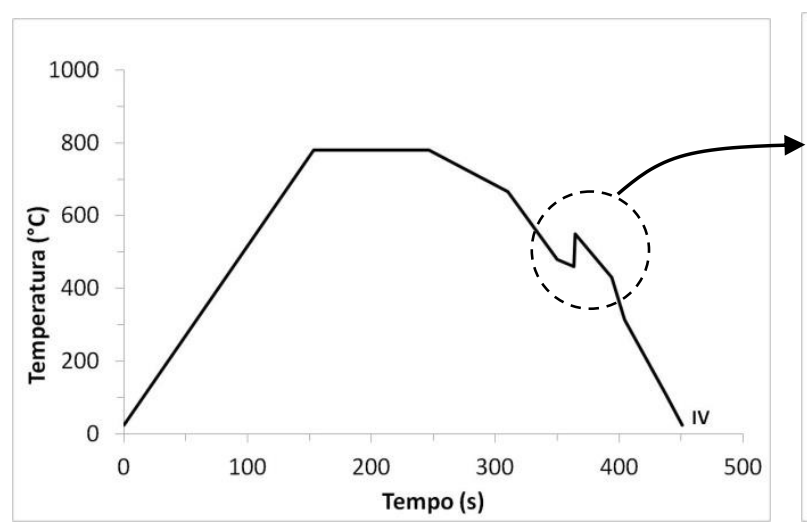

(a)

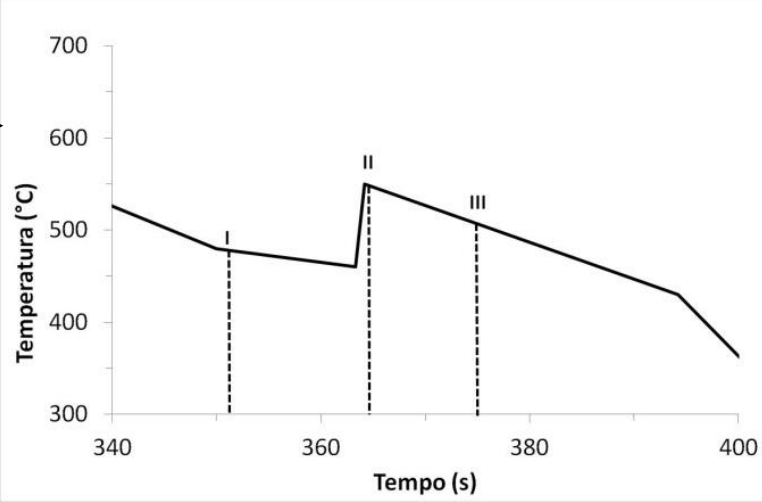

(b)

Figura 1. (a) Ciclo térmico completo e (b) detalhe na região de imersão no pote e tratamento térmico

Os revestimentos foram caracterizados por microscopia eletrônica de varredura com Field Emission Gun (MEV-FEG), microanálise via EDS (Energy Dispersive Spectroscopy) e espectroscopia de emissão ótica por descarga luminescente (GDOES - Glow Discharge Optical Emission Spectroscopy). A técnica GDOES realiza a análise química elementar da superfície de sólidos e também permite a determinação do perfil de concentração de elementos químicos ao longo da espessura do material (QDP-Quantitative Depth Profile). Durante a análise, a superfície é bombardeada continuamente por íons de argônio (sputtering), o que promove a remoção de átomos. Os átomos removidos são ionizados em um plasma e quando retornam ao estado de mais baixa energia emitem uma radiação com comprimento de onda $(\lambda)$ característico do elemento, que é medida pelo espectrômetro [5]. A correlação entre a intensidade da radiação e o tempo de bombardeamento permite a determinação do perfil de composição, isto é, das curvas concentração versus profundidade, normalmente expressas em termos de porcentagem em peso (\%p) por micrometros $(\mu \mathrm{m})$ [6].

A norma ISO 16.962 [6] estabelece a espessura do revestimento como sendo a profundidade na qual a concentração de Zn é igual a $50 \%$ do valor da concentração

\footnotetext{
* Contribuição técnica ao $51^{\circ}$ Seminário de Laminação - Processos e Produtos Laminados e Revestidos, 28 a 31 de outubro de 2014, Foz do Iguaçu, PR, Brasil.
} 


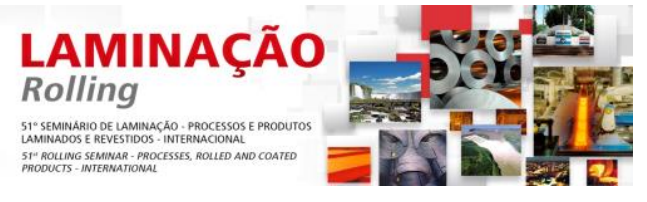

máxima deste elemento. Neste estudo, para maior praticidade, a espessura do revestimento foi estimada como sendo a profundidade na qual ocorre a interseção entre as curvas de concentração de Zn e Fe.

\section{RESULTADOS E DISCUSSÃO}

As análises das microestruturas de seção transversal dos revestimentos (Figuras 2 e 3) mostraram que a formação dos compostos intermetálicos na interface substrato/revestimento se iniciou ainda dentro do banho de zinco (Ponto I), para os teores de 0,09\% Al_ef e 0,14\% Al_ef, respectivamente, Figuras 2a e 2b. As análises via EDS dos cristais da interface (Figura 2c) mostraram picos de $\mathrm{Zn}$, Fe e Al, indicando a formação de compostos intermetálicos de Fe-Al (camada inibidora) e/ou de fases $\mathrm{Fe}-\mathrm{Zn}$, enquanto a análise da camada superior do revestimento apresentou apenas um pico intenso de zinco (Figura $2 \mathrm{~d}$ ), indicando se tratar de revestimento de zinco ainda não transformado.
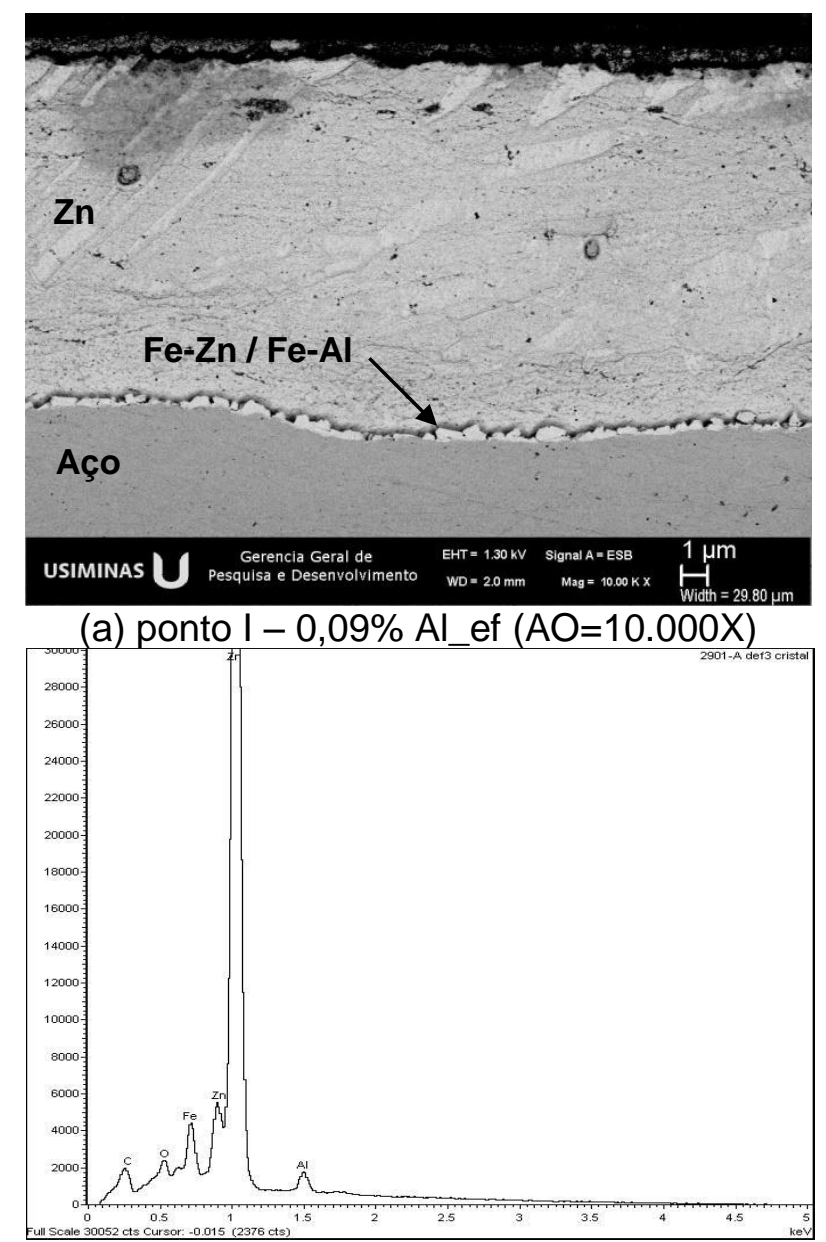

(c) EDS cristais na interface
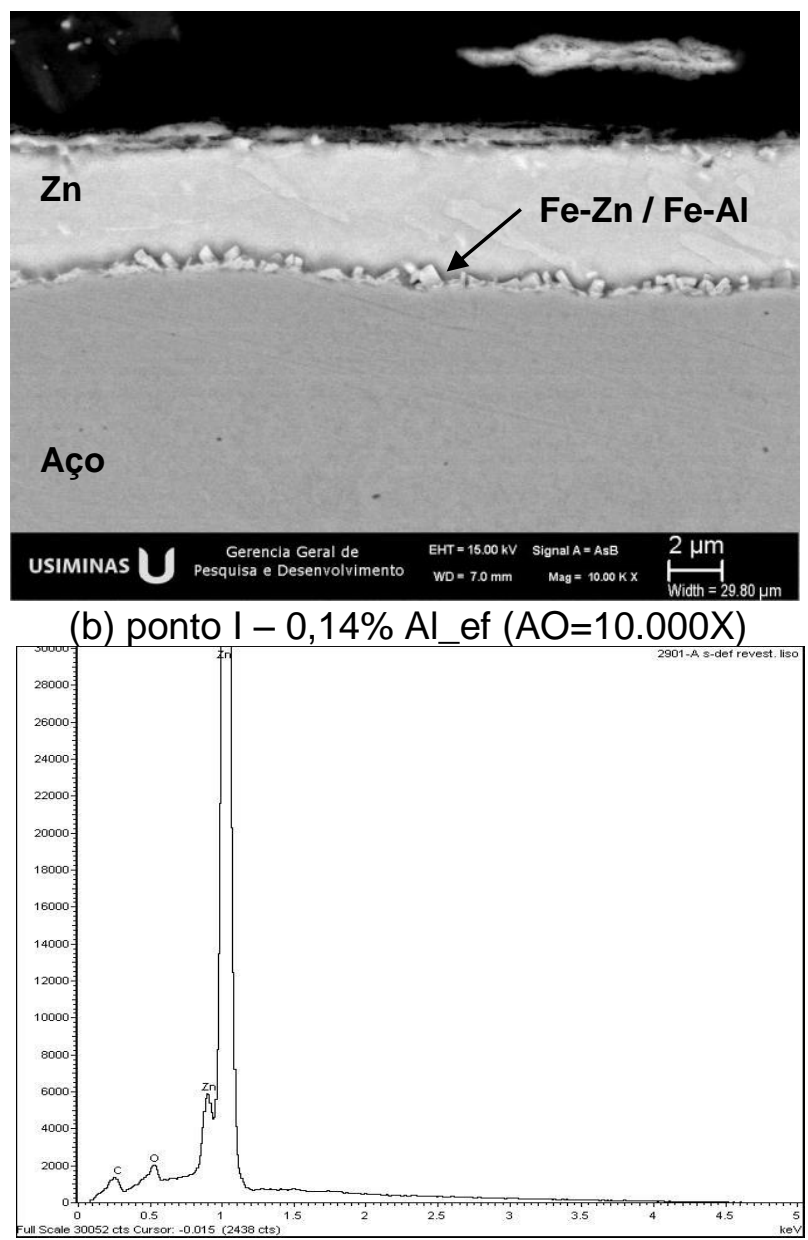

(d) EDS camada de $\mathrm{Zn}$

Figura 2. Fotomicrografias e espectros de EDS dos revestimentos formados no ponto I do ciclo de galvanização. ( $\mathrm{AO}=$ Aumento original).

Nas microestruturas dos revestimentos congelados após o aquecimento indutivo (pontos II e III, Figura 3), observou-se, claramente, a evolução da formação das fases $\mathrm{Fe}-\mathrm{Zn}$ da interface em direção à superfície com o aumento do tempo. No entanto, para os revestimentos produzidos com $0,14 \%$ Al_ef foi observado que a formação de cristais das fases Fe-Zn se deu com menor intensidade do que para os

* Contribuição técnica ao $51^{\circ}$ Seminário de Laminação - Processos e Produtos Laminados e Revestidos, 28 a 31 de outubro de 2014, Foz do Iguaçu, PR, Brasil. 


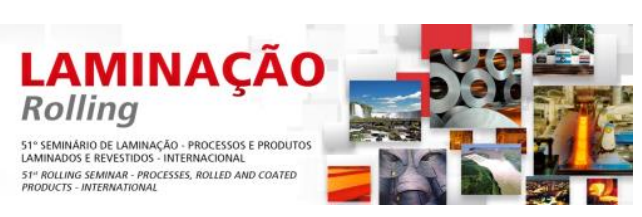

revestimentos com 0,09\% Al_ef (Figuras 3a, 3b, 3c e 3d). As análises via EDS dos cristais formados durante o tratamento térmico de galvannealing, em ambos os revestimentos, mostraram picos intensos de zinco e ferro, sem a presença de Al, indicando se tratar das fases Fe-Zn (Figura 3e).

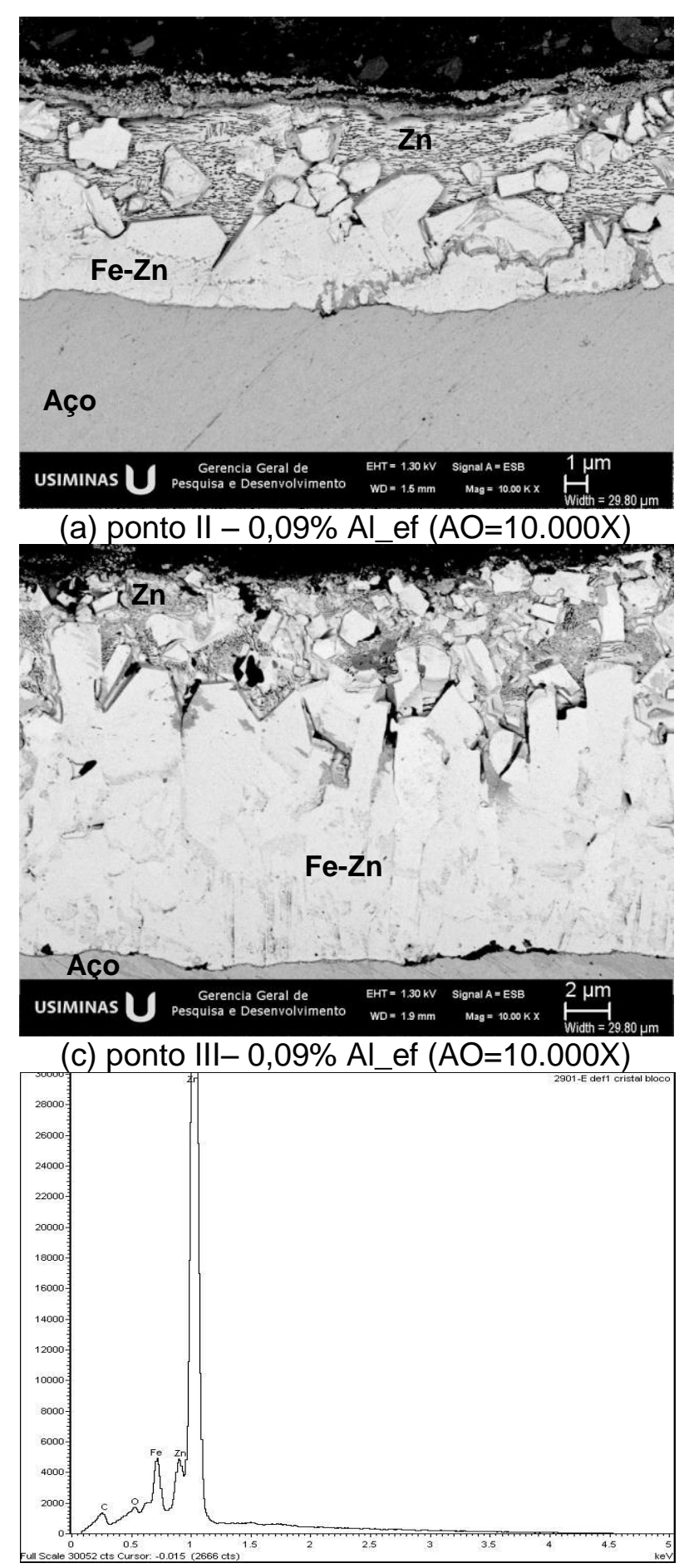

(e) EDS representativo das fases Fe-Zn

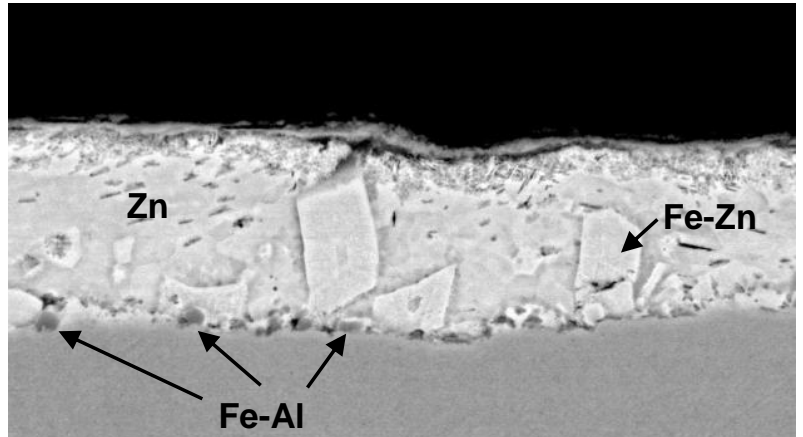

Aço

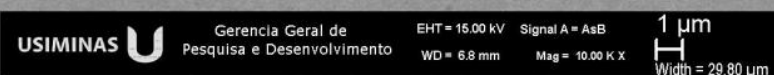

(b) ponto II - 0,14\% $\mathrm{Al}$ ef $(\mathrm{AO}=10.000 \mathrm{X})$

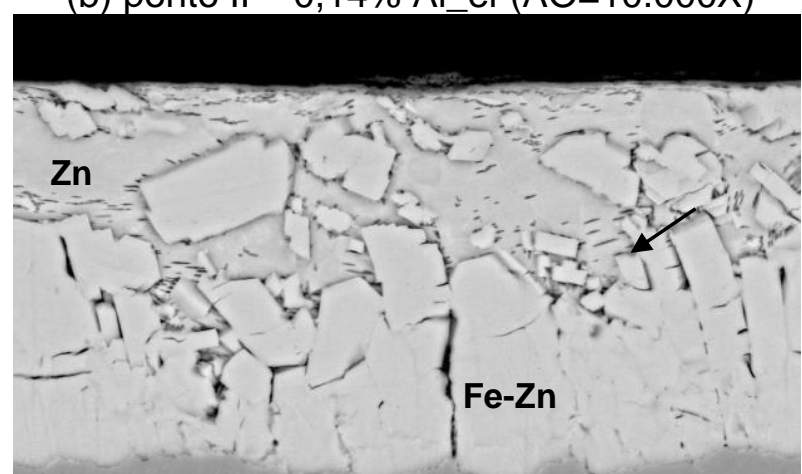

Aço

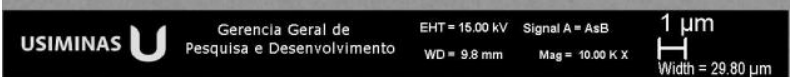

(d) ponto III - 0,14\% Al_ef $(\mathrm{AO}=10.000 \mathrm{X})$

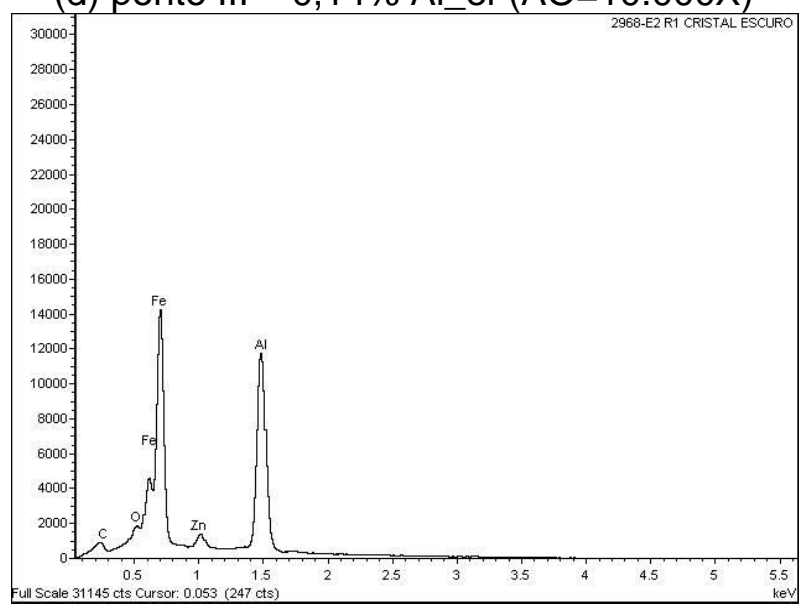

(f) EDS compostos Fe-Al na interface de (b) Figura 3: Fotomicrografias e espectros de EDS dos revestimentos formados nos pontos II e III do ciclo de galvanização. ( $\mathrm{AO}=$ Aumento original).

Já as análises via EDS de compostos presentes na interface do revestimento com maior alumínio efetivo mostraram picos de ferro e alumínio (Figura 3f), indicando se tratar de compostos Fe-Al, que podem ser associados com a formação da camada

* Contribuição técnica ao $51^{\circ}$ Seminário de Laminação - Processos e Produtos Laminados e Revestidos, 28 a 31 de outubro de 2014, Foz do Iguaçu, PR, Brasil. 




inibidora, e que não foram visualizados com a mesma intensidade no revestimento produzido com menor \%Al_ef. A presença dos compostos de Fe-Al na interface e a menor quantidade de cristais de Fe-Zn nos revestimentos com \%Al_ef mais alto, evidenciaram o papel do alumínio na formação da camada inibidora e no controle da velocidade de reação entre o ferro e o zinco.

Buscando esclarecer o comportamento do alumínio durante a formação do revestimento GA, foram realizadas análises via GDOES dos revestimentos produzidos na simulação (Figura 4). Foram avaliados os perfis de concentração ao longo da espessura do revestimento para os elementos $\mathrm{Fe}, \mathrm{Zn}$ e $\mathrm{Al}$ e, para melhor visualização, a concentração de alumínio foi multiplicada por cem vezes. Analisando comparativamente os perfis de composição química dos revestimentos produzidos com $0,09 \%$ Al_ef e $0,14 \%$ Al_ef nos mesmos pontos de congelamento das microestruturas, isto é, do Ponto I ao Ponto IV, observou-se diferenças na distribuição do alumínio entre os revestimentos, principalmente no início do processo de galvanização. Para os revestimentos com microestrutura congelada na região de imersão no banho (Ponto I, Figuras 4a e 4b), foram observados picos na concentração de Al na região da interface substrato/revestimento (região da interseção das curvas $\mathrm{Zn}$ e Fe), que caracterizam a formação da camada inibidora. No entanto, para o revestimento com 0,14\% Al_ef o pico de alumínio mostrou-se mais largo e intenso do que para o revestimento com $0,09 \%$ Al_ef, indicando a formação de uma camada de compostos Fe-Al mais contínua e/ou espessa na interface.

Para os revestimentos com microestrutura congelada durante o tratamento térmico de galvannealing, observou-se que logo após o aquecimento (ponto II, Figuras 4c e 4d) o alumínio tendeu a concentrar-se na interface revestimento/substrato. Neste estágio do processo de galvanização, o revestimento ainda se encontrava no estado líquido, o que facilitou a difusão. Este comportamento foi mais intenso para o revestimento produzido com $0,14 \%$ Al_ef e, provavelmente, está relacionado com a cinética mais rápida das reações $\mathrm{Fe}-\mathrm{Al}$ em comparação com as reações $\mathrm{Fe}-\mathrm{Zn}$. Acredita-se que o Al presente no revestimento fundido tenha migrado para a região da interface aonde a abundância de ferro vindo do substrato era maior. Com a evolução da formação das fases $\mathrm{Fe}-\mathrm{Zn}$, os compostos com alumínio migraram gradativamente da interface em direção à superfície e se distribuíram ao longo da espessura dos revestimentos (pontos III e IV, Figuras 4e, 4f, $4 \mathrm{~g}$ e 4h). Simultaneamente, foi observado o aumento da concentração de ferro ao longo do revestimento, evidenciando a formação das fases Fe-Zn. Em um estudo anterior dos mesmos autores [7], foi mostrado que o alumínio se difunde no revestimento em direção à superfície acompanhando a evolução das fases $\zeta\left(F e Z n_{13}\right)$ e $\delta\left(F e Z n_{7}\right)$.

Visando avaliar a qualidade superficial após o ciclo completo de galvanização (ponto IV, Figura 1a) foram realizadas análises visuais dos revestimentos. Nessas análises, identificou-se a existência de pequenas regiões com aspecto mais brilhante no revestimento GA, sendo que estas regiões aparentemente se formam mais no revestimento produzido com $0,14 \% \mathrm{Al}$ ef. As análises via MEV e os espectros via EDS característicos das regiões com aspecto brilhante são mostradas na figura 5 . Observou-se que nessas regiões os cristais das fases $\mathrm{Fe}-\mathrm{Zn}$ não estavam completamente formados, apresentando-se como uma camada fina, lisa e descontínua de revestimento (Figuras 5a e 5b).

\footnotetext{
* Contribuição técnica ao $51^{\circ}$ Seminário de Laminação - Processos e Produtos Laminados e Revestidos, 28 a 31 de outubro de 2014, Foz do Iguaçu, PR, Brasil.
} 


\section{LAMINAÇÃO \\ Rolling}

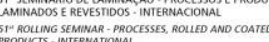
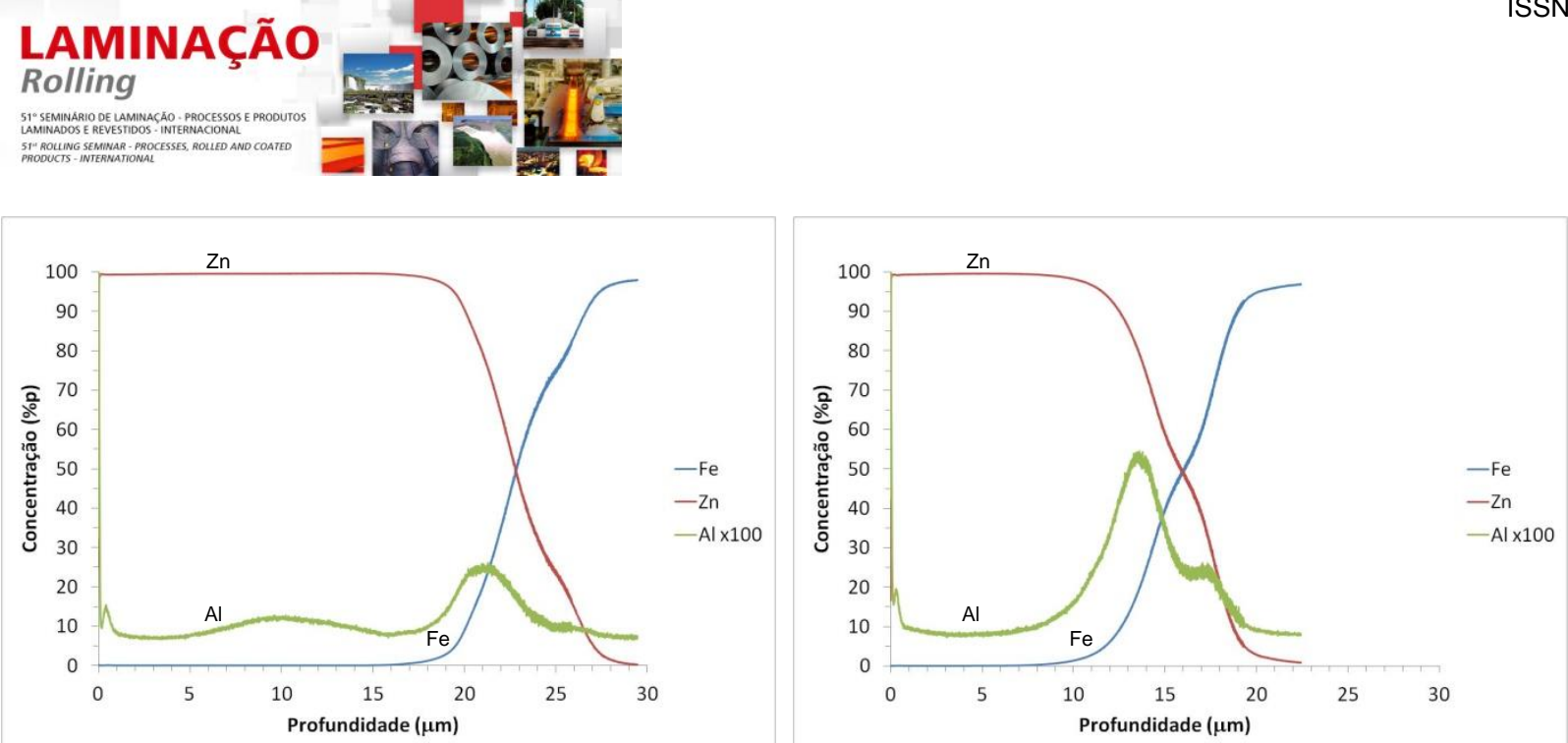

(a) ponto I-0,09\% Al_ef

(b) ponto I-0,14\% Al_ef

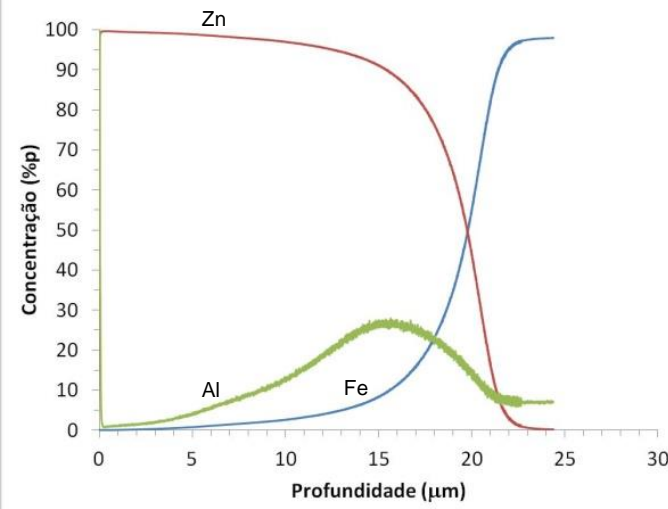

(c) ponto II - 0,09\% Al_ef
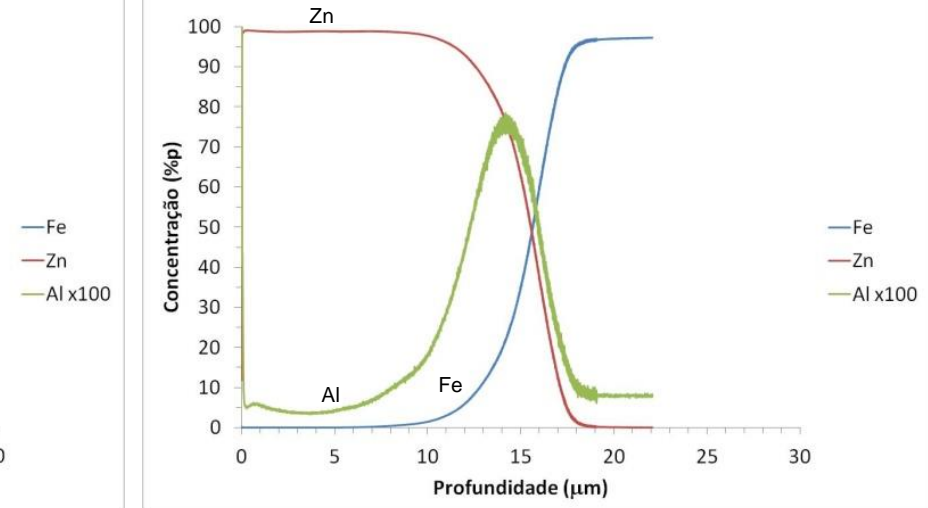

(d) ponto II - 0,14\% Al_ef

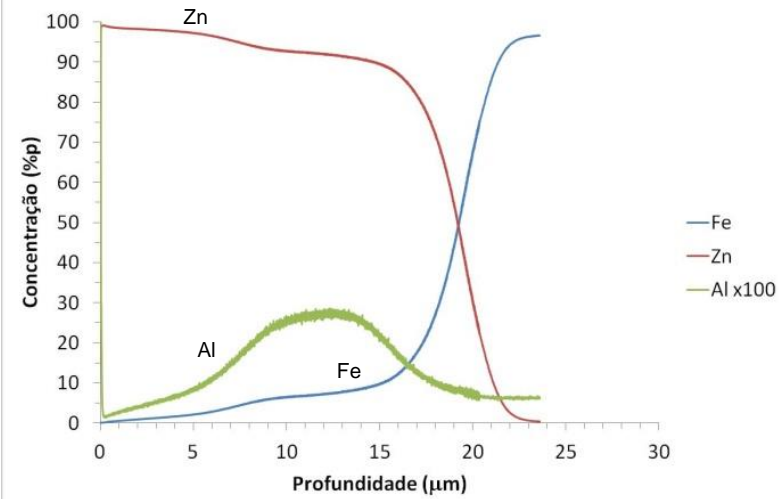

(e) ponto III - 0,09\% Al_ef

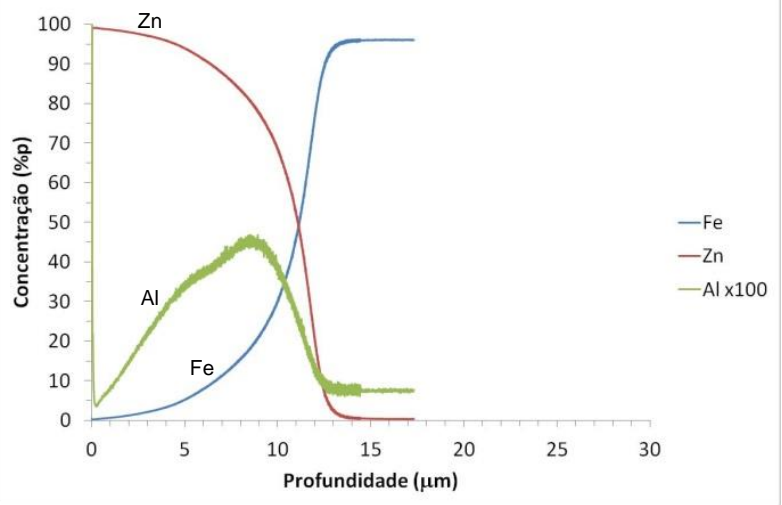

(f) ponto III - 0,14\% Al_ef
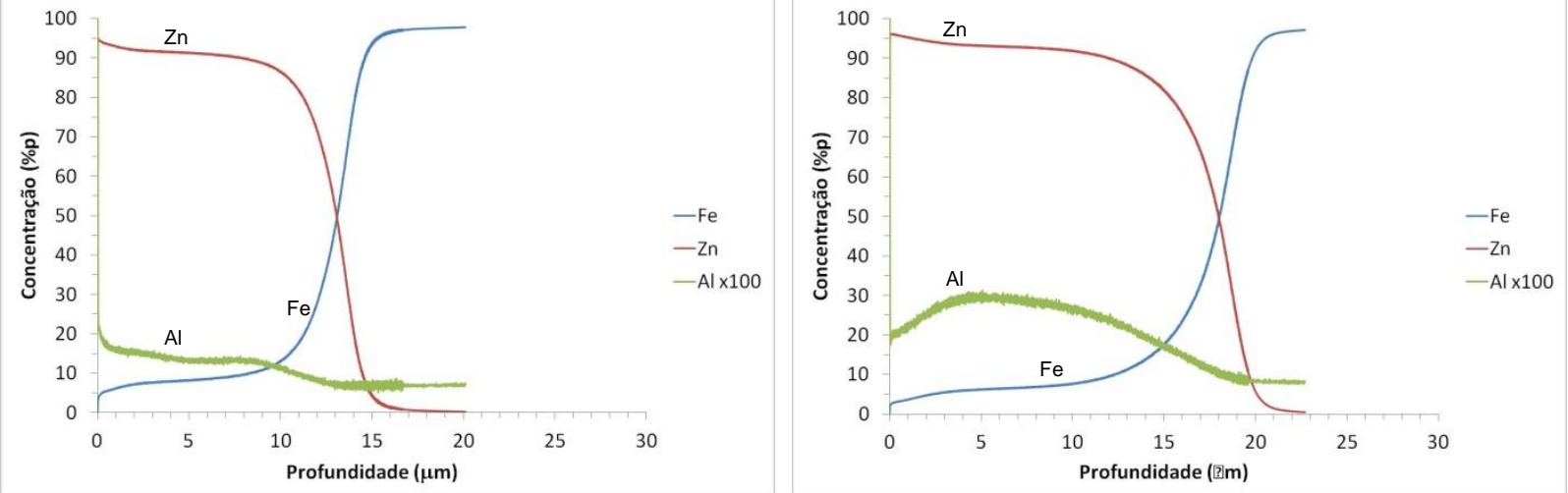

(g) ponto IV - 0,09\% Al_ef

(h) ponto IV - 0,14\% Al_ef

Figura 4: Perfis de composição química dos revestimentos nos pontos de I a IV via GDOES.

* Contribuição técnica ao $51^{\circ}$ Seminário de Laminação - Processos e Produtos Laminados e Revestidos, 28 a 31 de outubro de 2014, Foz do Iguaçu, PR, Brasil. 


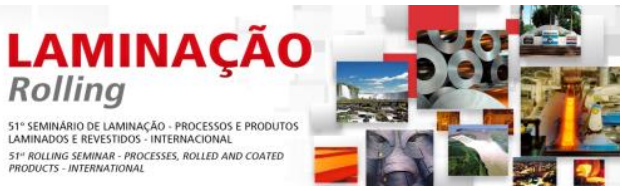
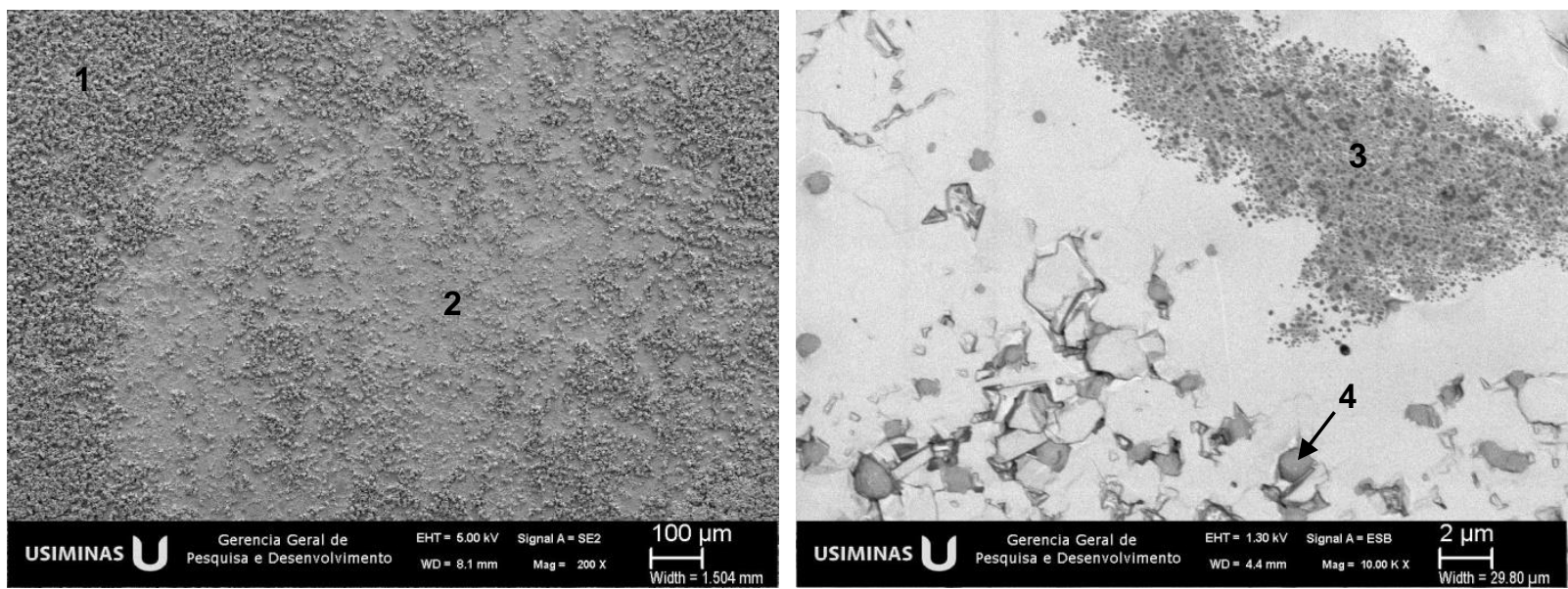

(a) Região brilhante no revestimento GA

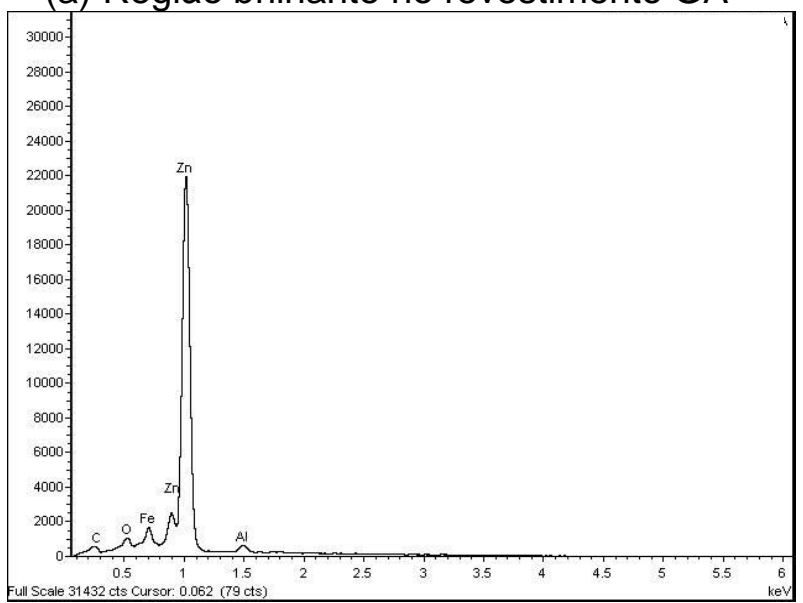

(b) Detalhe fundo da região brilhante

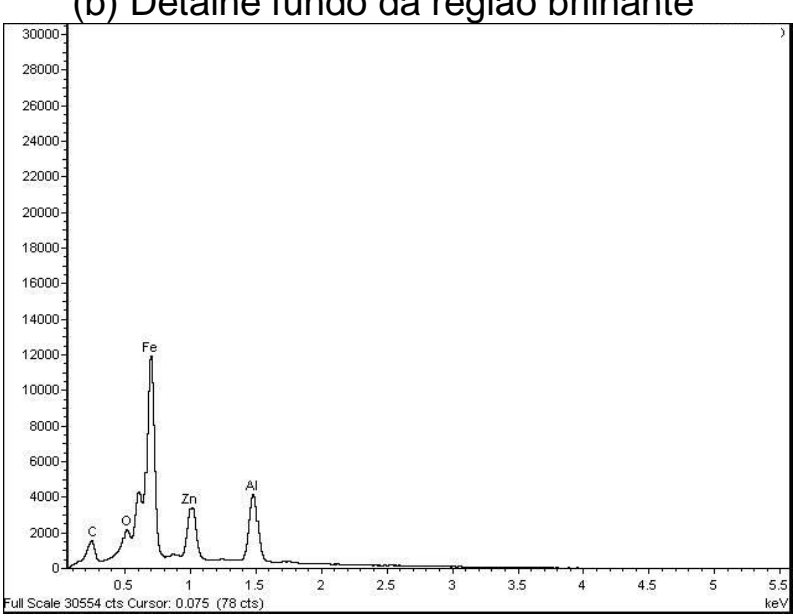

(c) EDS revestimento - região 1 em (a)
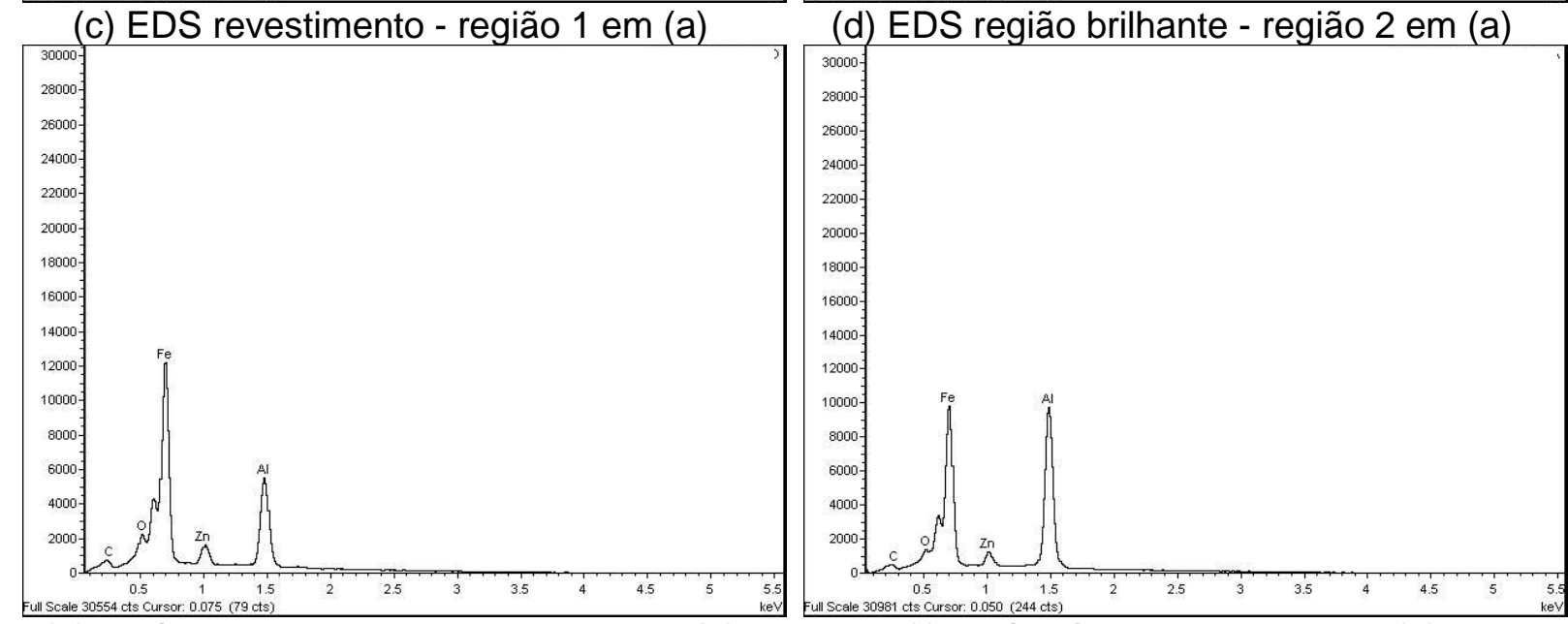

(e) EDS descontinuidade - região 3 em (b)

(f) EDS glóbulo - região 4 em (b)

Figura 5: Fotomicrografias e espectros de EDS representativos da região com aspecto brilhante nos revestimentos formados no ponto IV do ciclo de galvanização.

O espectro de EDS do revestimento (Figura 5c) mostrou picos de Zn e Fe, típicos dos revestimentos GA completamente transformados em fases Fe-Zn. Já o espectro da região brilhante (Figura $5 \mathrm{~d}$ ) apresentou picos intensos para o $\mathrm{Fe} \mathrm{e} \mathrm{Al,} \mathrm{e} \mathrm{um} \mathrm{pico}$ um pouco menos intenso para o $\mathrm{Zn}$, sugerindo uma maior influência do substrato, que pode ser atribuída a menor espessura da camada, e uma maior quantidade de compostos com Al na região.

O espectro de EDS das regiões de descontinuidade do revestimento (Figura 5e) apresentou picos intensos de ferro e alumínio, evidenciando a presença de

* Contribuição técnica ao $51^{\circ}$ Seminário de Laminação - Processos e Produtos Laminados e Revestidos, 28 a 31 de outubro de 2014, Foz do Iguaçu, PR, Brasil. 


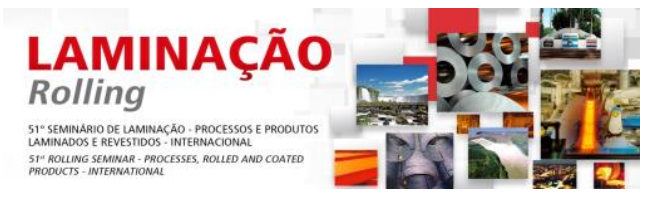

compostos $\mathrm{Fe}-\mathrm{Al}$, relacionados à formação de uma camada inibidora. Também foram observados glóbulos nas regiões de aspecto brilhante, cujos espectros de EDS confirmaram se tratar de compostos Fe-Al (Figura 5f). A identificação de compostos $\mathrm{Fe}-\mathrm{Al}$, na forma de camada e/ou de glóbulos, associada às regiões com aspecto brilhante, aponta a elevada concentração de alumínio localmente como uma das possíveis causas para a ocorrência desse fenômeno em revestimentos GA. A presença do alumínio em quantidade mais elevada pode ser considerada como uma causa provável para a transformação incompleta do zinco em fases $\mathrm{Fe}-\mathrm{Zn}$ durante o tratamento térmico de galvannealing, e consequentemente, para a ocorrência de regiões de aspecto brilhantes.

Assim, os resultados das simulações mostraram que o percentual de alumínio efetivo no banho exerce uma influência significativa na formação das fases Fe-Zn durante 0 tratamento térmico de galvannealing, sendo que quanto maior a concentração de Al maior a quantidade e compostos Fe-Al formados na interface substrato/revestimento e, consequentemente, menor a velocidade de formação das fases Fe-Zn que compõem o revestimento GA. Ficou evidenciado que a formação de regiões com aspecto brilhante está associada a um teor de alumínio mais elevado localmente, o que causou a transformação incompleta do Zn em fases Fe-Zn nessas regiões do revestimento durante o tratamento térmico de galvannealing. Como forma de se evitar a formação de regiões com aspecto brilhante, que podem impactar a qualidade superficial dos revestimentos GA, é recomendável se trabalhar com banho de Zn com um percentual de alumínio efetivo mais baixo.

\section{CONCLUSÃo}

A qualidade superficial dos revestimentos GA foi favorecida pela utilização de um percentual de alumínio efetivo mais baixo no banho de $\mathrm{Zn}$, isto é 0,09\% Al_ef. Este teor de Al favoreceu a cinética de formação das fases Fe-Zn durante o tratamento térmico de galvannealing e diminuiu a ocorrência de regiões com aspecto brilhante na superfície do revestimento, melhorando a qualidade da superfície.

\section{REFERÊNCIAS}

1 Barbosa AHA. Efeito das condições de tratamento de galvannealing na qualidade do revestimento galvanizado a quente "GA" formado sobre aços de alta resistência contendo P e B [Tese de Doutorado]: Universidade Federal de Minas Gerais; 2009.

2 Baril, E, L'Esperance, G, Boutin, E. Effect of process parameters on inhibition breakdown mechanisms during hot dip galvannealing. Galvatech'98 Conference Proceedings. 1998, 168-173.

3 Nakayama M, Kanamaru T, Numakumr Y. Effect of alloying conditions on phase growth behavior of galvannealed steel sheet. Defect and Diffusion Forum, 1993; 95-98: 661666.

4 Van Koesveld W, Lamberigts M, Van der Heiden A, Bordignon I. Coating microstructure assessment and control for advanced product properties of galvannealed if steels. Galvatech ' 95 Conference Proceedings. 1995; 343-355.

5 GLOW DISCHARGE DOT COM < www.glow-discharge.com > Acesso: abril, 2012.

6 ISO 16962-2005. Surface chemical analysis - Analysis of zinc and/or aluminium metallic coatings by glow-discharge optical-emission spectroscopy, ISO- International Organization for Standardization, 2005

7 Goulart-Santos S, Anício DF, Barbosa AHA. Estudo da distribuição de fases Fe-Zn em revestimentos galvannealed utilizando a técnica GDOES. Tecnologia em Metalurgia Materiais e Mineração. 2013; 10:254-261.

\footnotetext{
* Contribuição técnica ao $51^{\circ}$ Seminário de Laminação - Processos e Produtos Laminados e Revestidos, 28 a 31 de outubro de 2014, Foz do Iguaçu, PR, Brasil.
} 